\title{
MÁS ALLÁ DEL PAPEL DE LOS RECURSOS PERSONALES POSITIVOS EN EL BIENESTAR DE ESTUDIANTES UNIVERSITARIOS ESPAÑOLES: ¿IMPORTA LA INTELIGENCIA EMOCIONAL?
}

\author{
Nicolás Sánchez-Álvarez ${ }^{1}$, Lourdes Rey ${ }^{1}$, Natalio Extremera ${ }^{1}$ y Olivia D. Chang ${ }^{2}$ \\ ${ }^{1}$ Universidad de Málaga, España \\ ${ }^{2}$ Universidad de Michigan, EEUU
}

\section{Resumen}

Este estudio examina el papel de dos recursos personales positivos, optimismo y gratitud, como predictores del bienestar. Más allá de la capacidad predictiva del optimismo y la gratitud, se analiza el posible papel incremental de la inteligencia emocional en la predicción del bienestar. Los participantes fueron 350 estudiantes universitarios españoles (208 mujeres) con edades comprendidas entre los 18 y 30 años. Todos ellos respondieron de forma voluntaria y anónima a las escalas de gratitud, optimismo, inteligencia emocional, afectividad positiva y negativa, plenitud vital, y depresión. Los resultados de los análisis de correlación mostraron asociaciones positivas y significativas entre optimismo, gratitud e inteligencia emocional con afectividad positiva y plenitud, por el contrario, mostraron asociaciones negativas y significativas con la afectividad negativa y depresión. Asimismo, los resultados de los análisis de regresión jerárquica indicaron efectos explicativos significativos de la gratitud y el optimismo sobre la afectividad positiva y negativa, la plenitud, y la depresión. Más aún, la inteligencia emocional aportó varianza incremental predictiva a estos indicadores de bienestar positivo y negativo más allá de las variables sociodemográficas y estos recursos personales clásicos. Se discuten algunas implicaciones para comprender el papel de la inteligencia emocional como factor clave en el desarrollo del bienestar.

Palabras clave: Gratitud, Optimismo, Inteligencia Emocional, Depresión, Bienestar

\begin{abstract}
This study examines the role of two positive personal resources, optimism and gratitude, as predictors of well-being. Beyond the predictive role of optimism and gratitude, the potential incremental role of emotional intelligence in the prediction of different well -being outcomes was also analysed. The participants were 350 Spanish university students (208 females), aged between 18 and 30 years. All of them responded voluntarily and anonymously to the gratitude, optimism, emotional intelligence, positive and negative affectivity, flourishing, and depression scales. Correlational analysis showed significant and positive associations between optimism, gratitude, and emotional intelligence scores with positive affectivity and flourishing and negative and significant associations with negative affectivity and depression. Likewise, hierarchical regression analyses indicated significant predictive effects of gratitude and optimism on positive and negative affectivity, flourishing, as well as depression. Moreover, emotional intelligence added significant predictive utility in accounting for unique variance in all positive and negative well-being outcomes, beyond socio-demographics variables and other well-known classic personal resources. Some implications for the unique and genuine role of emotional intelligence as a key factor in the development of well-being are discussed.
\end{abstract}

Keywords: Gratitude, Optimism, Emotional Intelligence, Depression, Well-being

Correspondencia.

\section{Nicolás Sánchez-Álvarez \\ nsa@uma.es}

Universidad de Málaga, Facultad de Psicología.

Campus de Teatinos, 29071, Málaga 


\section{Introducción}

Cada vez cobra mayor importancia la presencia del bienestar en nuestras vidas (Hendriks et al., 2020), emergiendo en los últimos años una nueva área que examina aquellos recursos personales psicológicos que podrían favorecer el desarrollo y mantenimiento del bienestar (Peterson y Seligman, 2004). Diversos estudios han demostrado que algunos recursos personales no solo pueden actuar como mecanismos para disminuir los estados emocionales negativos y síntomas depresivos, sino que además aumentan estados de ánimo positivos y relacionados con el bienestar (Donaldson et al., 2019; Schutte y Malouff, 2019; White et al., 2019). En concreto, estos estudios han demostrado como las personas con niveles más elevados en ciertas habilidades y recursos positivos se adaptan mejor a las circunstancias negativas y son capaces de experimentar más sentimientos de bienestar (Sánchez-Álvarez et al., 2015; Thielmann et al., 2020). Por ejemplo, ciertos recursos personales son relevantes para la mejora de la salud mental, favorecen un correcto desarrollo y una mejor adaptación al medio (Hendriks et al., 2020). A este respecto, dos de los recursos personales y fortalezas más investigados en el campo de la psicología positiva, que han sido vinculados con el bienestar y fomentados en psicoterapia, son el optimismo y la gratitud (Sin y Lyubomirsky, 2009). Profundizar en el análisis de estos factores protectores de experiencias negativas y potenciadores de emociones positivas asociados al bienestar resulta crucial para el desarrollo de líneas terapéuticas y de fomento de un desarrollo personal óptimo (White et al., 2019).

\section{Bienestar}

Históricamente, en el estudio del bienestar, se ha priorizado la línea de investigación centrada en el tratamiento o la reducción del malestar y las experiencias negativas (Salanova, 2008; Seligman, 2002). Sin embargo, actualmente se ha incorporado el análisis del bienestar como un estado de plenitud y equilibrio psicológico, destacando la experimentación de placer y valoración positiva de experiencias vitales (Diener y Diener, 1995). La experimentación de bienestar consta de dos dimensiones, a saber, aspectos afectivos-emocionales, derivada de la comparación de las experiencias positivas con las situaciones de afectividad negativa, que muestran un balance de satisfacción experimentado. Además, el bienestar se compone de una dimensión cognitiva-valorativa que comprende la satisfacción vital, haciendo alusión a la capacidad de sentir satisfacción con toda la trayectoria de su vida (Cuadra y Florenzano, 2003; Diener et al., 2009). Estas dos dimensiones de bienestar, aunque distintas, parecen estar estrechamente relacionadas, dado que una persona que valora negativamente sus experiencias anímicas y reconoce con mayor frecuencia las emociones desagradables como el miedo, la ansiedad o la tristeza identificará su satisfacción con su vida como pobre o negativa, incrementando las posibilidades de desarrollar patologías como la depresión (Vázquez et al., 2009). Por otro lado, las personas que experimentan emociones agradables como la alegría, el cariño, o la sorpresa, evalúan su satisfacción vital como más positiva y enriquecedora, valorando sus vidas con una sensación de mayor plenitud (Seligman, 2011). Así pues, el bienestar y la salud mental se encontrarían en un continuo de dos extremos compuestos por bajos niveles de afectividad negativa y sintomatología asociada a la depresión, junto a la experimentación de altos niveles de afectividad positiva y una valoración positiva de plenitud vital (i.e., flourishing; Keyes, 2002). Los resultados obtenidos por la literatura científica que profundiza en el logro positivo del desarrollo del bienestar han mostrado que existen recursos positivos cuyas diferencias individuales pueden actuar como mecanismos de protección, para disminuir las experiencias emocionales negativas y aumentando mayores niveles de bienestar (Donaldson et al., 2019; Hendriks et al., 2020; Schutte y Malouff, 2019; White et al., 2019; Wingate et al., 2007).

Una población en la que sería particularmente valioso estudiar esas diferencias individuales en las variables psicológicas positivas son los estudiantes universitarios. De hecho, la vida universitaria representa una de las etapas críticas en el desarrollo vital caracterizada por altos niveles de estresores que afectan al bienestar (Beiter et al., 2015). Las nuevas y crecientes presiones académicas a las que se someten los universitarios, tales como las prácticas profesionales, trabajos grupales, exámenes, manejo del tiempo, o excesivo volumen de materias o contenidos por aprender pueden ser fuentes de estrés que afectan al bienestar de los estudiantes universitarios y pueden contribuir a deteriorar su salud mental (Keyes et al., 2012). Con el objetivo de ajustar los programas de orientación universitarios a las necesidades específicas de sus estudiantes, es importante comprender qué otros factores psicosociales de sus vidas, más allá de los estresores académicos externos, podrían estar implicados en la experimentación de los bajos niveles de bienestar y problemas de salud mental (Pool y Qualter, 2012). En este sentido, el optimismo, la gratitud y la inteligencia emocional representan recursos personales psicosociales positivos potencialmente notables que pueden estar asociados con mejores indicadores de salud mental y mayores niveles de bienestar entre los jóvenes universitarios.

\section{Optimismo}

Uno de los recursos positivos clásicos con capacidad para fomentar un funcionamiento psicológico positivo es el optimismo (Chang, 2009). El optimismo hace referencia a la capacidad personal de tener unas expectativas positivas hacia el futuro (Scheier y Carver, 1985). No es sorprendente que la investigación sobre el papel del optimismo en el 
bienestar muestre asociaciones con importantes indicadores de bienestar, incluyendo altos niveles de afectividad positiva, sentimiento de felicidad, y satisfacción vital (Chang et al., 2020). Asimismo, el optimismo se ha asociado con la ausencia de déficits de bienestar, incluyendo una disminución de experiencias de estados de ánimo negativas, afectividad negativa y síntomas depresivos (Andersson, 1996; Chang et al., 1997). Las personas optimistas informan una mayor experimentación de afecto positivo y menos emociones negativas, siendo capaces de hacer frente a situaciones estresantes, reduciendo la intensidad y frecuencia de los estados de ánimo negativos que conducen a la depresión y consecuentemente, incrementando la sensación percibida de bienestar (Carver y Scheier, 2014). Por lo tanto, el optimismo parece funcionar no sólo como un factor protector contra el desajuste psicológico, sino también como un potenciador de la salud mental que promueve otros aspectos importantes del ajuste positivo (Plomin et al., 1992).

\section{Gratitud}

De manera similar, la gratitud muestra unos mecanismos de acción análogos sobre la promoción del bienestar, mediante el aumento de las experiencias emocionales positivas y disminuyendo las negativas (Wood et al., 2010). Como rasgo, la gratitud se concibe como una disposición hacia la apreciación y el reconocimiento de actos de bondad, y la manifestación de expresiones de emociones positivas hacia sí mismo y hacia los demás (McCullough et al., 2002). Cabe destacar que esta fortaleza personal presenta una faceta interpersonal que favorece el funcionamiento social, aumentando la conexión con otras personas, lo que a su vez se asocia positivamente con el bienestar y la promoción de la salud mental (Froh et al., 2010). Por lo tanto, la gratitud podría tener una doble vía de efecto en el bienestar, por un lado, las personas agradecidas expresan su gratitud mostrando emociones positivas de manera prosocial, aumentando su felicidad. En segundo lugar, a su vez esta expresión de gratitud facilita los lazos sociales consolidando las relaciones interpersonales positivas que, de forma indirecta, aumentan la experimentación del bienestar emocional a través de dichas relaciones positivas (Algoe, 2012; Brannan et al., 2013). Numerosos estudios han establecido la conexión entre gratitud y bienestar, examinando sus relaciones positivas con indicadores positivos de salud mental (Davis et al., 2016; Sánchez-Álvarez et al., 2020; Wood et al., 2010).

\section{Inteligencia emocional}

Más allá del papel de estos dos recursos personales positivos en indicadores positivos y negativos de bienestar, existen otros constructos psicológicos que podrían estar influyendo en indicadores de bienestar y ajuste tales como la inteligencia emocional (Sánchez-Álvarez et al., 2016). La inteligencia emocional se conceptualiza como la habilidad para percibir y entender las emociones propias y ajenas, discriminar entre ellas, y usar la información emocional para guiar el procesamiento y las acciones de uno mismo (Mayer y Salovey, 1997). De esta forma, la inteligencia emocional se ha postulado como un elemento clave en el desarrollo del bienestar (Zeidner et al., 2012). Las emociones positivas y negativas influyen en nuestra forma de pensar y actuar, dirigen nuestra toma de decisiones y, por lo tanto, guían nuestro comportamiento (Lazarus, 2006). Como tal, no es sorprendente que la inteligencia emocional esté asociada con varios indicadores de bienestar positivos tales como la satisfacción vital, la afectividad positiva y la felicidad (Sánchez-Álvarez et al., 2015). La literatura científica ha mostrado que las personas con altos niveles de inteligencia emocional son más hábiles para afrontar el estrés y los problemas de la vida cotidiana, así como mantener mejores relaciones interpersonales y recibir mayor apoyo social (Zeidner et al., 2012). Por tanto, dado que los niveles de bienestar de las personas no pueden ser completamente explicados por sus niveles de apreciación de las cosas buenas y la percepción optimista de la vida, existen razones para creer que después de controlar estas influencias, la inteligencia emocional podría seguir involucrada en la predicción de indicadores de bienestar en estudiantes universitarios.

\section{Propósito del presente estudio}

Los estudios desarrollados para evaluar los efectos explicativos y potenciadores de los recursos humanos sobre el bienestar suelen focalizarse en el análisis de un recurso personal concreto. Debido a la aparición de enfoques más comprehensivos para entender de forma conjunta el impacto predictivo de distintos recursos personales en el desarrollo del bienestar, existe un aumento en las investigaciones dirigidas a descubrir los efectos explicativos, solapamientos conceptuales y valores incrementales de estos recursos personales (Chang, 2009; Extremera et al., 2020; SánchezÁlvarez et al., 2020). En definitiva, este estudio tuvo dos objetivos centrales: 1) evaluar el papel del optimismo y la gratitud como predictores de índices de bienestar en muestra universitaria; y 2) examinar el efecto incremental de la inteligencia emocional sobre el optimismo y la gratitud en la predicción del desarrollo del bienestar. En línea con estudios previos (p.ej., Bailey et al., 2007; Chang, 2009; Sánchez-Álvarez et al., 2015; Seligman et al., 2005; Wood et al., 2010), esperamos que tanto el optimismo, la gratitud, y la inteligencia emocional estén positivamente asociados con el afecto positivo y la plenitud, mientras que muestren asociaciones negativas con el afecto negativo y la depresión. Además, consistente con el planteamiento teórico y los distintos meta-análisis realizados que sugieren que la inteligencia emocional es un importante predictor del bienestar (Sánchez-Álvarez et al., 2016; Zeidner et al., 2012), esperamos que, más allá de la posible influencia potencial 
del optimismo y la gratitud, las habilidades de inteligencia emocional expliquen varianza adicional en los indicadores positivos y negativos de bienestar en estudiantes universitarios.

\section{Método}

\section{Participantes}

En este estudio participó una muestra de conveniencia de 350 estudiantes universitarios españoles (208 mujeres y 142 hombres) del sur de España. Las edades oscilaron entre los 18 y los 30 años. La edad media fue de 21,66 (DT = 3,72 ) años. Este estudio conto con un diseño descriptivo, exploratorio de corte transversal.

\section{Instrumentos}

Gratitude Questionnaire (GQ; McCullough et al., 2002). Este cuestionario es una medida de seis ítems de disposición al agradecimiento, y está diseñado para evaluar las diferencias individuales en la propensión para experimentar gratitud en la vida diaria. El rango de respuestas se evalúa en una escala Likert de siete puntos desde 1 (Totalmente en desacuerdo) a 7 (Totalmente de acuerdo). Los participantes completaron la versión al castellano de la escala (Magallares et al., 2018).

Life Orientation Test-Revised (LOT-R; Scheier et al., 1994). Esta escala evalúa el optimismo mediante una medida de diez ítems (cuatro ítems de relleno) sobre las expectativas generalizadas de resultados positivos y negativos. Se pide a los encuestados que califiquen el grado de acuerdo con cada uno de los ítems en una escala Likert de cinco puntos. En general, las puntuaciones más altas en el LOT-R son indicativas de un mayor optimismo disposicional. La versión española utilizada fue la adaptación por parte de Ferrando et al. (2002).

Wong and Law Emotional Intelligence Scale (WLEIS; Wong y Law, 2002). El WLEIS es una medida de inteligencia emocional de 16 ítems, compuesta por cuatro escalas que aprovechan diferentes dimensiones de la inteligencia emocional, denominadas, percepción de las emociones propias (p.ej., "Tengo una buena comprensión de mis propias emociones"), percepción de las emociones ajenas (p.ej., "Soy sensible a los sentimientos y emociones de los demás"), uso de las emociones (p.ej., "Siempre me digo a mí mismo que soy una persona competente"), y regulación de las emociones (p.ej., "Me puedo calmar fácilmente cuando me siento enfadado"). Se pide a los encuestados que califiquen los elementos en una escala tipo Likert de siete puntos, desde 1 (Totalmente en desacuerdo) hasta 7 (Totalmente de acuerdo). Las puntuaciones más altas en WLEIS son indicativos de una mayor inteligencia emocional. Los participantes completaron la versión al castellano de la escala (Extremera et al., 2019).
Positive and Negative Affect Scales (PANAS; Watson et al., 1988). Esta es una medida de autoinforme que incluye 20 ítems de los cuales diez ítems se refieren al afecto positivo (p.ej., estoy inspirado) y diez ítems que se refieren al afecto negativo (p.ej., me siento tenso), con formato de respuesta de tipo Likert con 5 opciones de respuesta que oscilan entre 1 (Muy poco o nada) a 5 (Siempre o casi siempre). Las puntuaciones más altas en las escalas afecto positivo y negativo indican una mayor afectividad positiva y negativa, respectivamente. La versión al castellano usada en el presente estudio posee altos niveles de fiabilidad y validez (Sandín et al., 1999).

Flourishing Scale (FS; Diener et al., 2010). Esta escala es una medida compuesta de ocho ítems que evalúa varios aspectos de las relaciones positivas, sentimientos de competencia y propósito en la vida. Los participantes indican su grado de acuerdo con cada ítem en una escala Likert de siete puntos, 1 (Muy en desacuerdo) a 7 (Muy de acuerdo). En general, las puntuaciones más altas en el flourishing indican una mayor plenitud. Todas las frases de los ítems se han redactado de forma positiva. La versión al castellano presenta una excelente fiabilidad (Ramírez-Maestre et al., 2017).

Beck Depression Inventory (BDI; Beck et al., 1988). Utilizada para la evaluación de la intensidad de los síntomas depresivos, esta escala consta de veintiún ítems que evalúan el estado de ánimo negativo, el pesimismo, la pérdida de peso, la fatiga, los pensamientos suicidas, la culpa y la sensación de fracaso. Estos son respondidos en una escala tipo Likert de cuatro puntos con un rango de respuesta desde 0 (No se me aplica) a 3 (Se me aplica mucho). La puntuación total varía de 0 a 63 . De esta forma, los resultados de esta prueba que reflejen puntuaciones altas implicarán una mayor presencia de síntomas depresivos. En este estudio se utilizó la versión española validada (Sanz y Vázquez, 1998).

\section{Procedimiento}

Se solicitó la participación de los estudiantes universitarios mediante un muestreo no probabilístico y de conveniencia durante las clases del grado de Psicología de una universidad andaluza. A todos los participantes se les proporcionó un consentimiento informado por escrito de acuerdo con la Declaración de Helsinki y las pautas éticas y se les informó que los datos del cuestionario se mantendrían estrictamente confidenciales.

\section{Análisis de datos}

Se utilizó el paquete estadístico SPSS 25 para calcular los estadísticos descriptivos, correlaciones, regresiones jerárquicas, y consistencia interna ( $\alpha$ de Cronbach). Se revisaron los supuestos de normalidad en la distribución de las puntuaciones, así como los posibles efectos de multicoli- 
nealidad y homocedasticidad. Los datos mostraron una distribución apropiada, así como una ausencia de multicolinealidad y valores atípicos. Como se puede observar en la Tabla 1, los valores de asimetría y curtosis mostraron un rango de puntuaciones apropiado (-2 y 2). El test de multicolinealidad (VIF) muestran valores entre 1 y 10 , por lo que no existe multicolinealidad. La prueba de homocedasticidad de Levene de varianzas por sexo muestra resultados no significativos, indicando igualdad de varianza y por lo tanto homocedasticidad.

\section{Resultados}

Medias, desviaciones estándar, correlaciones y consistencias internas

Las puntuaciones medias, desviaciones estándar, índices de correlaciones y la fiabilidad de las medidas se muestran en la Tabla 1. Las correlaciones entre optimismo, gratitud e inteligencia emocional mostraron asociaciones significativamente positivas con la afectividad positiva y plenitud, mientras que, por el contrario, mostraron asociaciones significativamente negativas con la afectividad negativa y la sintomatología depresiva. Los coeficientes de fiabilidad de todas las medidas del estudio mostraron una buena consistencia interna.

Tabla 1.

Índices de correlación de Pearson, puntuaciones medias (desviaciones estándar), y coeficientes de fiabilidad

\begin{tabular}{|c|c|c|c|c|c|c|c|}
\hline & 1 & 2 & 3 & 4 & 5 & 6 & 7 \\
\hline 1. Optimismo & - & & & & & & \\
\hline 2. Gratitud & 0,43 & - & & & & & \\
\hline 3. Inteligencia Emocional & 0,53 & 0,42 & - & & & & \\
\hline 4. Afecto Positivo & 0,50 & 0,38 & 0,50 & - & & & \\
\hline 5. Plenitud & 0,53 & 0,35 & 0,62 & 0,59 & - & & \\
\hline 6. Afecto Negativo & $-0,40$ & $-0,28$ & $-0,36$ & $-0,25$ & $-0,32$ & - & \\
\hline 7. Depresión & $-0,52$ & $-0,45$ & $-0,48$ & $-0,47$ & $-0,54$ & 0,34 & - \\
\hline M & 20,02 & 33,56 & 81,40 & 35,02 & 45,40 & 23,37 & 7,86 \\
\hline (DT) & $(4,38)$ & $(5,96)$ & $(14,92)$ & $(6,70)$ & $(7,72)$ & $(7,06)$ & $(8,43)$ \\
\hline Curtosis & 0,23 & 0,56 & $-0,21$ & 0,39 & 1,78 & $-0,27$ & $-1,62$ \\
\hline Asimetría & $-0,55$ & $-0,85$ & $-0,43$ & $-0,52$ & -1.31 & 0,41 & 1,42 \\
\hline F Levene & 0,30 & 0,64 & 0,45 & 0,74 & 0,38 & 0,39 & 0,55 \\
\hline VIF & 1,76 & 1,52 & 1,88 & 1,69 & 2,30 & 1,25 & 1,50 \\
\hline Alpha & 0,72 & 0,79 & 0,89 & 0,83 & 0,88 & 0,78 & 0,91 \\
\hline
\end{tabular}

Nota. $\mathrm{M}=$ Media; DT = Desviación Típica; VIF = Inflación de la Varianza de un factor (Variance Inflation Factor); $\mathrm{N}=350$.

Todos los índices de correlación tienen un nivel de significación $\mathrm{p}<0,001$. 


\section{Análisis de regresión}

Para examinar la capacidad incremental de la inteligencia emocional sobre el optimismo y la gratitud en la predicción de los índices de bienestar, se realizaron un conjunto de análisis de regresión jerárquica. Para cada uno de estos análisis de predicción, se insertaron las puntuaciones de los indicadores sociodemográficos (edad y género) en el Paso 1 como covariables, seguido de las puntuaciones que evalúan el optimismo y la gratitud en el Paso 2. Finalmente, se incluyó la puntuación de la inteligencia emocional en el Paso 3. Los resultados de los análisis de regresión jerárquica para predecir los índices de bienestar se presentan en la Tabla 2. Tal como aparece en dicha tabla, con respecto a nuestros indicadores positivos de bienestar, más allá de las variables sociodemográficas, la gratitud $(\beta=0,20, p<$ $0,001)$ y el optimismo $(\beta=0,40, p<0,001)$ explicaron un $28 \%$ de la varianza de la afectividad positiva, mientras que la inteligencia emocional $(\beta=0,29, p<0,001)$ incrementaba la predicción en un $6 \%$. En el siguiente análisis de regresión jerárquica se evaluaron los efectos predictivos sobre la plenitud, donde la gratitud $(\beta=0,39, p<0,001)$ y el optimismo $(\beta=0,36, p<0,001)$ explicaron un $42 \%$ de la varianza explicada, mientras que la inteligencia emocional $(\beta$ $=0,39, p<0,001)$ incrementaba la predicción en un $10 \%$. Por otro lado, con respecto a los indicadores de bienestar negativo, al evaluar los efectos predictivos en la afectividad negativa, la gratitud $(\beta=-0,14, p<0,001)$ y el optimismo $(\beta=-0,34, p<0,001)$ explicaron un $18 \%$ de la varianza explicada, mientras que la inteligencia emocional $(\beta=$ $0,17, p=0,004)$ incrementaba la predicción en un $2 \%$. Por último, con respecto a la depresión, la gratitud $(\beta=-0,28, p$ $<0,001)$ y el optimismo $(\beta=-0,39, p<0,001)$ explicaron un $33 \%$ de la varianza explicada, mientras que la inteligencia emocional $(\beta=-0,22, p<0,001)$ incrementaba la predicción en un $4 \%$.

Tabla 2.

Resultados de análisis de regresión sobre los indicadores de bienestar

\begin{tabular}{|c|c|c|c|c|c|}
\hline & $\beta$ & $R^{2}$ & $\Delta R^{2}$ & $F$ & $p$ \\
\hline \multicolumn{6}{|l|}{ Afectividad Positiva } \\
\hline Paso 1 & & 0,00 & - & 1,20 & $\mathrm{p}=0,300$ \\
\hline Sexo & $-0,05$ & & & & \\
\hline Edad & 0,05 & & & & \\
\hline Paso 2 & & 0,28 & 0,28 & 35,09 & $\mathrm{p}<0,001$ \\
\hline Gratitud & $0,20 * * *$ & & & & \\
\hline Optimismo & $0,40 * * *$ & & & & \\
\hline Paso 3 & & 0,34 & 0,06 & 36,42 & $\mathrm{p}<0,001$ \\
\hline Inteligencia Emocional & $0,29 * * *$ & & & & \\
\hline \multicolumn{6}{|l|}{ Plenitud } \\
\hline Paso 1 & & 0,00 & - & 1,91 & $\mathrm{p}=0,149$ \\
\hline Sexo & 0,04 & & & & \\
\hline Edad & 0,06 & & & & \\
\hline Paso 2 & & 0,42 & 0,42 & 63,85 & $\mathrm{p}<0,001$ \\
\hline Gratitud & $0,39 * * *$ & & & & \\
\hline Optimismo & $0,36 * * *$ & & & & \\
\hline Paso 3 & & 0,52 & 0,10 & 76,61 & $\mathrm{p}<0,001$ \\
\hline Inteligencia Emocional & $0,39 * * *$ & & & & \\
\hline \multicolumn{6}{|l|}{ Afectividad Negativa } \\
\hline Paso 1 & & 0,00 & - & 1,29 & $\mathrm{p}=0,275$ \\
\hline Sexo & 0,08 & & & & \\
\hline Edad & 0,03 & & & & \\
\hline Paso 2 & & 0,18 & 0,18 & 19,82 & $\mathrm{p}<0,001$ \\
\hline Gratitud & $-0,14 * *$ & & & & \\
\hline Optimismo & $-0,34 * * *$ & & & & \\
\hline Paso 3 & & 0,20 & 0,02 & 17,91 & $\mathrm{p}=0,004$ \\
\hline Inteligencia Emocional & $-0,17 * *$ & & & & \\
\hline \multicolumn{6}{|l|}{ Depresión } \\
\hline Paso 1 & & 0,00 & - & 0,39 & $\mathrm{p}=0,677$ \\
\hline Sexo & 0,01 & & & & \\
\hline Edad & $-0,04$ & & & & \\
\hline Paso 2 & & 0,33 & 0,33 & 43,91 & $\mathrm{p}<0,001$ \\
\hline Gratitud & $-0,28 * * *$ & & & & \\
\hline Optimismo & $-0,39 * * *$ & & & & \\
\hline Paso 3 & & 0,38 & 0,04 & 40,26 & $\mathrm{p}<0,001$ \\
\hline Inteligencia Emocional & $-0,22 * * *$ & & & & \\
\hline
\end{tabular}

$\mathrm{N}=350$. Nota: $* * * \mathrm{p}<0,001, * * \mathrm{p}<0,01, * \mathrm{p}<0,05$ 


\section{Discusión}

El aumento de investigaciones sobre el impacto de los recursos positivos personales han permitido expandir nuestro conocimiento sobre los mecanismos clave en el desarrollo del bienestar (Hendriks et al., 2020). Sin embargo, la mayoría de estos estudios evalúan de forma individual la importancia de estos recursos positivos, lo que no permite evaluar los efectos sumativos de diversos recursos sobre ambos componentes, positivos y negativos, del bienestar. Acorde con la teoría del desarrollo de las emociones positivas de Fredrickson (2004), diversos recursos personales tales como el optimismo, la gratitud, o la inteligencia emocional impulsarían la resiliencia psicológica disminuyendo la experimentación de emociones negativas y fomentando la aparición de emociones de carácter positivo. Nuestros hallazgos confirman la relación significativa del optimismo, la gratitud y la inteligencia emocional con los indicadores afectivos positivos y negativos, así como con la sintomatología depresiva y la sensación de plenitud vital en estudiantes universitarios. Más aun, estos resultados confirman que los tres recursos personales muestran efectos predictivos significativos con cada uno de los indicadores de bienestar examinados.

En línea con la literatura, nuestros resultados muestran que el optimismo, la gratitud, y la inteligencia emocional son factores promotores asociados a múltiples indicadores de bienestar en estudiantes universitarios (Chang et al., 2020; Sánchez-Álvarez et al., 2015, 2020; Seligman, 2011). En concreto, estos resultados sugieren que aquellos estudiantes universitarios que tienen mayores expectativas positivas hacia el futuro tienden a experimentar una menor frecuencia de emociones negativas, así como experimentar un mayor número de emociones positivas, al mismo tiempo que indican tener menor sintomatología depresiva y una mayor sensación de plenitud con sus vidas (Seligman, 2011). En la misma línea, la gratitud muestra un patrón idéntico de asociaciones, acorde con la literatura (Davis et al., 2016; Sánchez-Álvarez et al., 2020; Wood et al., 2010). Es decir, los estudiantes universitarios más agradecidos tienden a experimentar más emociones positivas y menos negativas, así como un menor índice de sintomatología asociada a la depresión, mientras que muestran mayores niveles de plenitud con sus vidas. Estos resultados están en consonancia con investigaciones previas que sugieren un vínculo único y causal entre la capacidad de apreciar lo positivo y minimizar aquellas cuestiones negativas y el fomento del bienestar en general (Wood et al., 2010). Finalmente, nuestro estudio muestra que la inteligencia emocional también sigue el mismo patrón de relaciones positivas con la afectividad positiva y la plenitud, así como relaciones negativas con la afectividad negativa y la sintomatología depresiva
(Sánchez-Álvarez et al., 2015; Zeidner y Olnick-shemesh, 2010). Es decir, los estudiantes universitarios con mayores habilidades para el procesamiento de la información emocional muestran unos niveles más elevados de bienestar en general.

En conjunto, estos hallazgos sugieren que, en la predicción de indicadores tanto positivos como negativos del bienestar, la combinación de distintos recursos personales como son el optimismo, la gratitud, y la inteligencia emocional mejora la predicción del bienestar (Chang et al., 1997; Chang et al., 2009; Sánchez-Álvarez et al., 2020; Scheier y Carver, 1985). Por otro lado, los hallazgos obtenidos de validez incremental significativa de la inteligencia emocional en el bienestar señalan que parte de la varianza en la predicción de los indicadores de bienestar están condicionados por la gestión de las habilidades emocionales. Estos hallazgos son consistentes con nuestra hipótesis y muestran la capacidad genuina e idiosincrática de las habilidades emocionales, más allá de otros recursos personales clásicos, en la explicación, mantenimiento y desarrollo del bienestar en universitarios. Tal como se aprecia, la capacidad incremental de la inteligencia emocional fue mayor para los indicadores positivos (6\% para afectividad positiva y $10 \%$ para plenitud de varianza incremental) que para los indicadores negativos (2\% para afectividad negativa y $4 \%$ para depresión de varianza incremental), sugiriendo que la inteligencia emocional, más allá del efecto de otros recursos clásicos, podría estar más asociada con el fomento del bienestar universitario que con la reducción del malestar. Además, en términos generales, la capacidad explicativa de los tres recursos positivos analizados fue mayor para los indicadores positivos de bienestar, redundando en la idea de que estos recursos podrían tener más relación con el desarrollo y mantenimiento de aspectos positivos del bienestar universitario que con la reducción de los mismos. No obstante, la varianza explicada osciló, en general, entre el $20 \%$ y el $52 \%$, poniendo de manifiesto que tanto la gestión emocional como las expectativas positivas hacia el futuro y la capacidad de reconocer los actos de bondad hacia los demás, son elementos claves en el desarrollo del bienestar universitario

En conclusión, nuestros hallazgos subrayan al menos dos implicaciones importantes para las intervenciones dirigidas a fomentar el bienestar en poblaciones universitarias. Primero, promover el optimismo y la gratitud puede ser una forma potencial de fomentar el aumento de emociones positivas en detrimento de las negativas, disminuyendo la sintomatología depresiva y aumentando el sentimiento de plenitud (Carver y Scheier, 2014; Seligman, 2011; Vázquez et al., 2009). En segundo lugar, nuestro estudio identificó que las habilidades emocionales tienen un valor incremental único añadido al optimismo y la gratitud en la predicción de los distintos indicadores de bienestar, este hallazgo 
podría servir como base para las intervenciones de orientación universitaria. De hecho, los estudiantes universitarios corren un alto riesgo de sufrir estados de ánimo negativo, ansiedad y depresión los cuales, en parte, dependen de su exposición continua a múltiples factores de estrés académicos y sociales (Beiter et al., 2015), destacando la necesidad de intervenciones y programas preventivos que traten estos problemas de salud mental y falta de bienestar universitario (Pool y Qualter, 2012). Dado que la triada positiva compuesta por el optimismo, la gratitud, y la inteligencia emocional parecen ser aspectos importantes para conseguir una sensación de bienestar de los estudiantes universitarios, los servicios de orientación universitaria podrían centrarse en fomentar la formación de estos recursos personales como un camino viable para proteger a los estudiantes de los resultados psicológicos negativos, así como promover su bienestar positivo de los alumnos durante los años universitarios (Peterson y Seligman, 2004; White et al., 2019). Además, estos programas preventivos basados en recursos personales para estudiantes universitarios tendrían el potencial, no sólo de reducir indicadores de malestar y fomentar el bienestar, sino que indirectamente influir sobre otros indicadores académicos tales como la tasa de abandono, las calificaciones universitarias, las relaciones interpersonales y la cohesión de grupo, entre otros. Futuras investigaciones en esta línea deberían explorar el efecto de estos recursos personales en dichos indicadores de resultados universitarios y ajuste académico.

Aunque esta investigación tiene una serie de puntos fuertes, se deben considerar varias limitaciones, como basarse en datos auto-informados en una muestra de conveniencia de estudiantes universitarios españoles. Al mismo tiempo, el diseño de este estudio fue transversal, por lo que no permite inferir relaciones causales a través del tiempo. Los estudios futuros deben explorar y generalizar estos hallazgos utilizando un diseño aleatorio con nominaciones de pares de la relación personal con muestras de otros grupos culturales, así como diseños longitudinales que permitan establecer una temporalidad en cada etapa de los mecanismos de acción entre variables.

A pesar de estas limitaciones, este estudio proporciona apoyo empírico sobre el papel que el optimismo, la gratitud y la inteligencia emocional juegan como importantes predictores de la afectividad positiva y negativa, así como de la sintomatología depresiva y los niveles de plenitud vital en muestra de universitarios españoles, y destaca la necesidad de incorporar actividades para fomentar estos recursos personales psicosociales en los programas de prevención/ intervención como un potencial medio para el desarrollo óptimo del ajuste psicológico y el bienestar universitario.

\section{Referencias}

Algoe, S.B. (2012). Find, remind, and bind: the functions of gratitude in everyday relationships. Social and Personality Psychology Compass, 6(6), 455-469. https://doi.org/10.1111/j.1751-9004.2012.00439.x

Andersson, G. (1996). The benefits of optimism: A metaanalytic review of the life orientation test. Personality and Individual Differences, 21(5), 719-725. https://doi.org/10.1016/0191-8869(96)00118-3

Bailey, T.C., Eng, W., Frisch, M.B. y Snyder, C.R. (2007). Hope and optimism as related to life satisfaction. Journal of Positive Psychology, 2(3), 168-175. https://doi.org/10.1080/17439760701409546

Beck, A.T., Steer, R.A. y Garbin, M.G. (1988). Psychometric properties of the Beck Depression Inventory: Twenty-five years of evaluation. Clinical Psychological Review, 8, 77-100. https:// doi.org/10.1016/0272-7358(88)90050-5

Beiter, R., Nash, R., McCrady, M., Rhoades, D., Linscomb, M., Clarahan, M. y Sammut, S. (2015). The prevalence and correlates of depression, anxiety, and stress in a sample of college students. Journal of Affective Disorders, 173, 90-96. https:// doi.org/10.1016/j.jad.2014.10.054

Brannan, D., Biswas-Diener, R., Mohr, C.D., Mortazavi, S. y Stein, N. (2013). Friends and family: A crosscultural investigation of social support and subjective well-being among college students. Journal of Positive Psychology, 8(1), 65-75. https:// doi.org/10.1080/17439760.2012.743573

Carver, C.S. y Scheier, M.F. (2014). Dispositional optimism. Trends in Cognitive Sciences, 18(6), 293299. https://doi.org/10.1016/j.tics.2014.02.003

Chang, E.C., Maydeu-Olivares, A. y D’Zurilla, T.J. (1997). Optimism and pessimism as partially independent constructs: Relationship to positive and negative affectivity and psychological well-being. Personality and Individual Differences, 23(3), 433-440. https://doi.org/10.1016/S0191-8869(97)80009-8

Chang, E.C. (2009). An examination of optimism, pessimism, and performance perfectionism as predictors of positive psychological functioning in middleaged adults: Does holding high standards of performance matter beyond generalized outcome expectancies? Cognitive Therapy and Research, 33(3), 334-344. https://doi.org/10.1007/s10608-008-92159

Chang, E.C., Chang, R. y Sanna, L.J. (2009). Optimism, pessimism, and motivation: Relations to adjustment. 
Social and Personality Psychology Compass, 3(4), 494-506. https://doi.org/10.1111/j.17519004.2009.00190.x

Chang, E.C., Yi, S., Liu, J., Kamble, S.V., Zhang, Y., Shi, B., Ye, Y., Fang, Y., Cheng, K., Xu, J., Shen, J., Li, M. y Chang, O.D. (2020). Coping behaviors as predictors of hedonic well-being in asian indians: Does being optimistic still make a difference? Journal of Happiness Studies, 21(1), 289-304. https:// doi.org/10.1007/s10902-019-00087-w

Cuadra L.H. y Florenzano U.R. (2003). El bienestar subjetivo: Hacia una psicología Positiva. Revista de Psicología, 12(1), 83-96. https://doi.org/10.5354/07190581.2003 .17380

Davis, D.E., Choe, E., Meyers, J., Wade, N., Varjas, K., Gifford, A., Quinn, A., Hook, J.N., Van Tongeren, D.R., Griffin, B.J. y Worthington, E.L. (2016). Thankful for the little things: A meta-analysis of gratitude interventions. Journal of Counseling Psychology, 63(1), 20-31. https://doi.org/10.1037/ cou0000107

Diener, E. y Diener, M. (1995). Personality processes and individual cross-cultural correlates of life satisfaction and self-esteem. Journal of Personality, 68(4), 653-663. https://doi.org/10.1007/978-90-481-2352$0 \_4$,

Diener, E., Wirtz, D., Biswas-Diener, R., Tov, W., KimPrieto, C., Choi, D. y Oishi, S. (2009). Assessing well-being. Social Indicators Research, 39, 247266. https://doi.org/10.1007/978-90-481-2354-4

Diener, E., Wirtz, D., Tov, W., Kim-Prieto, C., Choi, D. won, Oishi, S. y Biswas-Diener, R. (2010). New well-being measures: Short scales to assess flourishing and positive and negative feelings. Social Indicators Research, 97(2), 143-156. https:// doi.org/10.1007/s11205-009-9493-y

Donaldson, S.I., Lee, J.Y. y Donaldson, S.I. (2019). evaluating positive psychology interventions at work: A systematic review and meta-analysis. International Journal of Applied Positive Psychology, 4(3), 113134. https://doi.org/10.1007/s41042-019-00021-8

Emmons, R.A. y Mishra, A. (2011). Why gratitude enhances well-being: what we know, what we need to know. En M. S. Kennon, T. B. Kashdan y M. F. Steger (Eds.), Designing Positive Psychology: Taking Stock and Moving Forward (pp. 248-262). Oxford University Press.

Extremera, N., Rey, L. y Sánchez-Álvarez, N. (2019). Validation of the Spanish version of the Wong Law
Emotional Intelligence Scale (WLEIS-S). Psicothema, 31(1), 94-100. https://doi.org/10.7334/ psicothema2018.147

Extremera, N., Sánchez-Álvarez, N. y Rey, L. (2020). Pathways between ability emotional intelligence and subjective well-being: Bridging links through cognitive emotion regulation strategies. Sustainability, 12 (5), 2111. https://doi.org/10.3390/su12052111

Ferrando, P.J., Chico, E., Tous, J.M. y Tous, M. (2002). Propiedades psicométricas del test de optimismo Life Orientation Test. Psicothema, 14(3), 673-680. https://doi.org/0214 - 9915

Fredrickson, B.L. (2004). The broaden-and-build theory of positive emotions. Philosophical Transactions of the Royal Society B: Biological Sciences, 359(1449), 1367-1377. https://doi.org/10.1098/rstb.2004.1512

Froh, J.J., Bono, G. y Emmons, R. (2010). Being grateful is beyond good manners: Gratitude and motivation to contribute to society among early adolescents. Motivation and Emotion, 34(2), 144-157. https:// doi.org/10.1007/s11031-010-9163-z

Hendriks, T., Schotanus, M. y Aabidien, D. (2020). the efficacy of multi-component positive psychology interventions: A systematic review and meta-analysis of randomized controlled trials. Journal of Happiness Studies, 21(1), 357-390. https:// doi.org/10.1007/s10902-019-00082-1

Keyes, C.L. (2002). The mental health continuum: From languishing to flourishing in life. Journal of Health and Social Behavior, 43(2), 207-222. https:// doi.org/10.2307/3090197

Lazarus, R.S. (2006). Emotions and interpersonal relationships: Toward a person-centered conceptualization of emotions and coping. Journal of Personality, 74(1), 9-46. https://doi.org/10.1111/j.14676494.2005.00368.x

Magallares, A., Recio, P. y Sanjuan, P. (2018). Factor structure of the Gratitude Questionnaire in a Spanish sample. Spanish Journal of Psychology, 21, E53. https://doi.org/10.1017/sjp.2018.55

Mayer, J.D. y Salovey, P. (1993). The intelligence of emotional intelligence. Intelligence, 17(4), 433-442. https://doi.org/10.1016/0160-2896(93)90010-3

Mayer, J.D. y Salovey, P. (1995). Emotional intelligence and the construction and regulation of feelings. Applied and Preventive Psychology, 4(3), 197-208. https://doi.org/10.1016/S0962-1849(05)80058-7

McCullough, M.E., Emmons, R.A. y Tsang, J.A. (2002). 
The grateful disposition: A conceptual and empirical topography. Journal of Personality and Social Psychology, 82(1), 112-127. https:// doi.org/10.1037/0022-3514.82.1.112

Peterson, C. y Seligman, M.E. (2004). Character Strengths and Virtues: A Handbook and Classification (Vol. 1). Oxford University Press.

Plomin, R., Scheier, M.F., Bergeman, C.S., Pedersen, N.L., Nesselroade, J.R. y McClearn, G.E. (1992). Optimism, pessimism and mental health: A twin/ adoption analysis. Personality and Individual Differences, 13(8), 921-930. $\mathrm{https} / /$ doi.org/10.1016/0191-8869(92)90009-E

Pool, L.D. y Qualter, P. (2012). Improving emotional intelligence and emotional self-efficacy through a teaching intervention for university students. Learning and individual Differences, 22(3), 306-312.

Ramírez-Maestre, C., Correa, M., Rivas, T., LópezMartínez, A.E., Serrano-Ibáñez, E.R. y Esteve, R. (2017). Psychometric characteristics of the Flourishing Scale-Spanish Version (FS-SV). The factorial structure in two samples: Students and patients with chronic pain. Personality and Individual Differences, 117, 30-36. https://doi.org/10.1016/ j.paid.2017.05.035

Rey, L., Sánchez-Álvarez, N. y Extremera, N. (2018). Spanish Gratitude Questionnaire: Psychometric properties in adolescents and relationships with negative and positive psychological outcomes. Personality and Individual Differences, 135, 173-175. https:// doi.org/10.1016/j.paid.2018.07.005

Salanova, M. (2008). Organizaciones saludables: Una aproximación desde la psicología positiva. Psicología Positiva Aplicada, 403-427.

Sánchez-Álvarez, N., Extremera, N. y Fernández-Berrocal, P. (2016). The relation between emotional intelligence and subjective well-being: A meta-analytic investigation. The Journal of Positive Psychology, 11(3), 276-285. https:// doi.org/10.1080/17439760.2015.1058968

Sánchez-Álvarez, N., Extremera, N., Rey, L., Chang, E.C. y Chang, O.D. (2020). Optimism and gratitude on suicide risk in Spanish adults: Evidence for doubling up or doubling down? Journal of Clinical Psychology, 76(10), 1882-1892. https:// doi.org/10.1002/jclp. 22962

Sandín, B., Chorot, P., Lostao, L., Joiner, T., Santed, M. y Valiente, R. (1999). Escalas PANAS de afecto positivo y negativo: validación factorial y convergencia transcultural. Psicothema, 11(1), 37-51.

Sanz, J. y Vázquez, C. (1998). Fiabilidad, validez y datos normativos del Inventario para la Depresión de Beck. Psicothema, 10(2), 303-318. https:// doi.org/7467-13170-1

Scheier, M.F. y Carver, C.S. (1985). Optimism, coping, and health: Assessment and implications of generalized outcome expectancies. Health Psychology, 4 (3), 219-247. https://doi.org/10.1037/02786133.4.3.219

Scheier, M.F., Carver, C.S. y Bridges, M.W. (1994). Distinguishing optimism from neuroticism (and trait anxiety, self-mastery, and self-esteem): A reevaluation of the Life Orientation Test. Journal of Personality and Social Psychology, 67(6), 10631078. https://doi.org/10.1037/0022-3514.67.6.1063

Schutte, N.S. y Malouff, J.M. (2019). The impact of signature character strengths interventions: A meta-analysis. Journal of Happiness Studies, 20(4), 1179-1196. https://doi.org/10.1007/s10902-0189990-2

Seligman, M.E. (2002). Positive Psychology, Positive Prevention, and Positive Therapy. En C.R. Snyder y S.J. Lopez (Eds.), Handbook of Positive Psychology (pp. 3-9). Oxford University Press.

Seligman, M.E. (2011). Flourish: A visionary new understanding of happiness and well-being. Policy, 27 (3), 60-61.

Seligman, M.E., Steen, T.A., Park, N. y Peterson, C. (2005). Positive psychology progress: Empirical validation of interventions. American Psychologist, 60(5), 410-421. https://doi.org/10.1037/0003066X.60.5.410

Sin, N.L. y Lyubomirsky, S. (2009). Enhancing well-being and alleviating depressive symptoms with positive psychology interventions: A practice-friendly metaanalysis. Journal of Clinical Psychology, 65(5), 467 -487. https://doi.org/10.1002/jclp.20593

Thielmann, I., Spadaro, G. y Balliet, D. (2020). Personality and prosocial behavior: A theoretical framework and meta-analysis. Psychological Bulletin, 146(1), 30-90. http://dx.doi.org/10.1037/bul0000217

Vázquez, C., Hervás, G., Rahona, J.J. y Gómez, D. (2009). Psychological well-being and health: Contributions of positive psychology. Annuary of Clinical and Health Psychology, 5, 15-27.

Watson, D., Clark, L.A. y Tellegen, A. (1988). Development and validation of brief measures of positive 
and negative affect: The PANAS scales. Journal of Personality and Social Psychology, 54(6), 10631070. https://doi.org/10.1037/0022-3514.54.6.1063

White, C.A., Uttl, B. y Holder, M.D. (2019). Meta-analyses of positive psychology interventions: The effects are much smaller than previously reported. PloS One, 14(5), e0216588. https://doi.org/10.1371/ journal.pone. 0216588

Wingate, L.R., Burns, A.B., Gordon, K.H., Perez, M., Walker, R.L., Williams, F.M. y Joiner, T.E. (2007). Suicide and positive cognitions: positive psychology applied to the understanding and treatment of suicidal behavior. Cognition and Suicide: Theory, Research, and Therapy, 261-283. https:// doi.org/10.1037/11377-012

Wong, C.S. y Law, K.S. (2002). The effects of leader and follower emotional intelligence on performance and attitude: An exploratory study. Leadership Quarterly, 13(3), 243-274. https://doi.org/10.1016/S1048 $-9843(02) 00099-1$

Wood, A.M., Froh, J.J. y Geraghty, A.W.A. (2010). Gratitude and well-being: A review and theoretical integration. Clinical Psychology Review, 30(7), 890905. https://doi.org/10.1016/j.cpr.2010.03.005

Wood, A.M. y Joseph, S. (2010). The absence of positive psychological (eudemonic) well-being as a risk factor for depression: A ten year cohort study. Journal of Affective Disorders, 122(3), 213-217. https:// doi.org/10.1016/j.jad.2009.06.032

Yu, E.A. y Chang, E.C. (2021). Relational meaning in life as a predictor of interpersonal well-being: A prospective analysis. Personality and Individual Differences, 168, 110377. https://doi.org/10.1016/ j.paid.2020.110377

Zeidner, M., Matthews, G. y Roberts, R.D. (2012). The emotional intelligence, health, and well-being nexus: What have we learned and what have we missed? Applied Psychology: Health and WellBeing, 4(1), 1-30. https://doi.org/10.1111/j.17580854.2011.01062.x

Zeidner, M. y Olnick-shemesh, D. (2010). Emotional intelligence and subjective well-being revisited. Personality and Individual Differences, 48(4), 431-435. https://doi.org/10.1016/j.paid.2009.11.011 